\title{
Balance de fases usando colonia de hormigas
}

\author{
Alejandro Garcés-Ruiz*, Mauricio Granada-Echeverri*, Ramón A. Gallego-R.*\$ \\ * Grupo de Investigación en Planeamiento de Sistemas Eléctricos \\ Facultad de Ingeniería Eléctrica, Universidad Tecnológica de Pereira. \\ §e-mail: ragr@utp.edu.co
}

(Recibido: Septiembre 5 de 2005 - Aceptado: Noviembre 29 de 2005)

\begin{abstract}
Resumen
Los sistemas de distribución operan de forma desbalanceada debido a sus características de configuración y naturaleza de sus cargas. Es aconsejable disminuir este desbalance ya que una operación en estas condiciones presenta un mayor nivel de pérdidas a las obtenidas en un sistema balanceado. En este documento, se presenta una aplicación de la metodología de colonia de hormigas al problema de balance de fases mostrando sus principales características y resolviendo un ejemplo de prueba.
\end{abstract}

Palabras Clave: Balance de Fases, Sistemas de Distribución, Desequilibrio nodal, Colonia de Hormigas, Metodologías Heurísticas.

\begin{abstract}
Distribution systems operate in unbalanced form because of their configuration characteristics and nature of their loads. It is advisable to increase the balance level of the system because an operation in unbalanced conditions presents a greater level of losses. In this document, an ants colony methodology is presented and applied to a phase balance problem; likewise, the main characteristics of this methodology are shown by solving a test system.
\end{abstract}

Keywords: Phase balance, distribution systems, unbalanced loads, ants colony, heuristic methodology.

\section{Introducción}

En los sistemas de distribución es común encontrar cargas y configuraciones desbalanceadas, lo cual no sólo ocasiona aumento en las pérdidas sino también riesgos de sobre-tensiones y efectos negativos sobre la calidad del servicio. Así mismo las elevadas corrientes en el neutro inherentes al desbalance pueden ocasionar disparo en las protecciones así como una limitación adicional en la capacidad de las líneas dada por el límite térmico del neutro. Este artículo presenta la formulación matemática del problema de balance de fases y describe de forma general la metodología de colonia de hormigas aplicándola, finalmente, a un ejemplo de prueba.

El problema de balance de fases consiste en encontrar 
una configuración de conexión de cargas, en función de las fases, que reduzca de forma óptima el nivel de pérdidas en el sistema de distribución. Este problema puede ser planteado para un punto de operación en la curva de carga (potencia máxima) o para un horizonte de planeamiento. El planeamiento resulta de gran interés en sistemas con escasos recursos, ya que las medidas tomadas permanecen vigentes durante el período de tiempo considerado, lo cual constituye una gran diferencia con los sistemas altamente automatizados. Así, el balance de fases es una medida correctiva aplicada al sistema de distribución que propenda reducir las pérdidas y que involucra costos de inversión relativamente bajos si se comparan con los involucrados en otras medidas como la instalación de capacitores o FACTS y cambio de conductores, entre otras. Por esta razón, implementar programas de balanceo de fases en las empresas de distribución de energía eléctrica es una alternativa viable, que debe ser considerada no sólo para la reducción de pérdidas técnicas, sino también, para aumentar la calidad, seguridad y confiabilidad del servicio, así como para reducir las inversiones adicionales en la aplicación de otras medidas correctivas posteriores tales como la reconfiguración y la compensación de reactivos.

Un balance perfecto en las corrientes y voltajes del sistema generalmente es difícil de obtener dada las características de las cargas y los alimentadores existentes en el sistema de distribución, entre las cuales cabe destacar las configuraciones monofásicas y trifásicas tanto para líneas como para cargas. Los sistemas monofásicos de tres hilos cuentan con dos fases de un sistema trifásico más un neutro y los monofásicos de dos hilos se presentan cuando se utilizan dos fases del sistema trifásico o una fase y el neutro. En sistemas trifásicos la distribución puede realizarse con tres o cuatro hilos, donde este último caso se presenta cuando se cuenta con un neutro [1]. Toda esta diversidad en configuraciones, sumado a que las cargas a su vez pueden tener configuraciones trifásicas $o$ monofásicas, implica un modelado trifásico de todos los elementos del sistema, así como el desarrollo de un flujo de potencia trifásico que permita avaluar las pérdidas del sistema y obtener índices de desbalance nodal del mismo[2].

En Zhu [3] se muestra un algoritmo que utiliza una aproximación lineal del problema con solución única y asume modelos de carga de corriente constante, por lo cual no es necesario implementar un flujo de carga exacto, aunque en sistemas de gran tamaño se requiere de un elevado esfuerzo computacional. En la actualidad existen métodos, como el de Puntos Interiores, que han mostrado ser más eficientes que el método tradicional Simplex y que pueden ayudar a reducir significativamente el esfuerzo computacional invertido en la solución de problemas lineales de gran tamaño. Sin embargo, debe tenerse en cuenta que se tomarían decisiones sobre el modelo lineal, que es un modelo aproximado y sólo es adecuado en puntos cercanos al punto de operación alrededor del cual se realiza el análisis.

Zhu también propone en [4] un algoritmo de balance de fases aplicando el método de optimización combinatorial denominado Recocido Simulado (Simulated Annealing). En este método, la función objetivo es de tipo no-lineal, la cual es más adecuada y considera los costos de realizar un cambio en cada carga. Cuando se intenta resolver el problema no lineal para un sistema de distribución de gran tamaño, surge el denominado fenómeno de explosión combinatorial y el problema fácilmente puede convertirse en un problema NP-completo, lo que implica que el tiempo requerido para evaluar todas las opciones resultantes puede resultar prohibitivo (décadas, cientos o miles de años). En estos casos se renuncia, por lo menos teóricamente, a encontrar la solución óptima del problema y se buscan soluciones subóptimas de alta calidad. Los métodos combinatoriales, y en particular las técnicas evolutivas, tienen la capacidad de encontrar soluciones de buena calidad explorando pocos subespacios del espacio solución. De otro lado, debido a las consideraciones adicionales que se pueden presentar en la administración del sistema eléctrico, es más conveniente contar con un conjunto de alternativas subóptimas de buena calidad, que con una solución única.

\section{Formulación del problema}

El objetivo del balance de fases es reducir el nivel de desbalance de corrientes por las líneas y de esta manera disminuir pérdidas y corrientes por el neutro, por lo cual, la mejor solución para el problema de 
balance de fases es aquella configuración de conexión de cargas que no produzca corrientes de secuencia cero. Sin embargo, como se dijo anteriormente, obtener una solución con estas características es poco probable dadas las características de un sistema típico de distribución:

$\checkmark$ Presencia de cargas y ramales monofásicos.

$\checkmark$ Dificultad para hacer cambios a nivel secundario.

$\checkmark$ Presencia de cargas combinadas.

Por lo tanto, la función objetivo del modelo matemático consiste en encontrar la configuración que minimice las pérdidas en mayor porcentaje, considerando el costo de la energía, un horizonte de planeamiento y unas restricciones de operación.

El modelo matemático del problema para un horizonte de planeamiento a mediano plazo que involucre la curva de carga discretizada en periodos de tiempo que pueden ser variables (ver figura 1), es el siguiente:

$$
\begin{aligned}
& \text { Min } C_{E} \sum_{t=1}^{T_{m}} T_{t} \cdot \operatorname{Perdidas}\left(V_{(3 \phi, t)}, \theta_{(3 \phi, t)}\right) \\
& P_{(3 \phi, t)}=f_{P}\left(V_{(3 \phi, t)}, \theta_{(3 \phi, t)}\right) \\
& Q_{(3 \phi, t)}=f_{Q}\left(V_{(3 \phi, t)}, \theta_{(3 \phi, t)}\right) \\
& V_{\min }<V_{(3 \phi, t)}<V_{\max } \\
& H_{i} \in\{1, . .6\} \\
& H_{i} \leq\left. 3 \quad \forall i\right|_{i=\{\text { Carga Motriz }\}}
\end{aligned}
$$

Donde, $\mathbf{C}_{\mathrm{E}}$ representa el costo de la energía, Tt es el tiempo de la configuración de carga $t$ y $\mathrm{Tm}$ es el número de periodos en que se discretiza la curva. $\mathrm{H}$ es una variable que toma valores enteros de 1 a 6 y que corresponden a las posibles conexiones que se pueden realizar como se muestra en la tabla 1 . La función objetivo mostrada en (1) tiene en cuenta las pérdidas de energía del sistema a lo largo del horizonte de planeamiento. De considerarse sólo un punto de la curva de carga (valor pico), la variable Tm tiene valor de 1 y la función objetivo podría ser planteada en función de potencia únicamente. Así mismo, en el caso general, la función objetivo puede tener en cuenta tanto pérdidas de potencia como pérdidas de energía, así como el costo de realizar cada uno de los cambios. Las restricciones (2) y (3) definen el flujo de carga trifásico para cada uno de los Tm períodos a considerar; la restricción (4) tiene en cuenta los niveles de tensión máximo y mínimo de cada uno de los nodos del sistema; en (5) la variable $\mathrm{H}$ define la nueva configuración de las cargas según las posibles combinaciones mostradas en la tabla 1 y la restricción (6) considera el cambio de secuencia en cargas motrices. El problema presenta alta explosión combinatorial por lo cual la aplicación de técnicas heurísticas es apropiada.

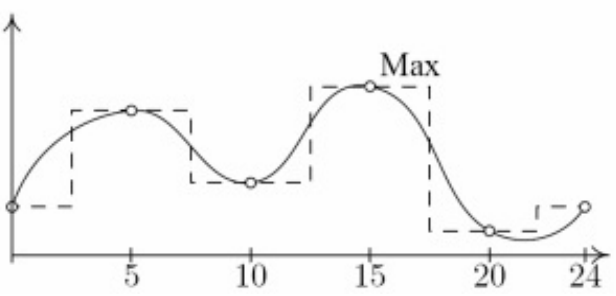

Figura 1: Curva de Carga discretizada en 5 intervalos

El balance de fases necesariamente debe considerar el modelamiento trifásico de los elementos característicos del sistema de distribución, como son: líneas aéreas y subterráneas, cargas, bancos de capacitores, reguladores, cogeneradores y equivalente externo de la red, entre otros. Todos estos elementos son integrados a través de un flujo de carga trifásico que permite evaluar la función objetivo, la cual depende directamente de las pérdidas del sistema, como se muestra en (1). El proceso de optimización que reduce las pérdidas totales del sistema reacomodando las cargas entre las diferentes fases, es guiado por índices de sensibilidad que miden el grado de desbalance total del sistema. De esta forma, se puede presentar el caso en que una carga tenga una configuración altamente desbalanceada, pero desde el punto de vista del desbalance global sea benéfica puesto que disminuye las pérdidas totales del sistema.

Cuando el balance de fases se realiza para un período de tiempo determinado, se debe considerar una curva de carga discretizada, como se muestra en la figura 1, y las modificaciones propuestas por la metodología 
resultan ser aquellas que presentan las menores pérdidas para todo el período. Es necesario utilizar una codificación que permita identificar todos los posibles tipos de conexión que se pueden realizar en el sistema de distribución y que adicionalmente facilite la incorporación de la técnica de optimización propuesta. Para ello, se debe tener en cuenta que sólo se realizarán cambios entre fases, en el secundario de cada transformador, de la totalidad de la carga conectada, es decir, la carga se considera indivisible. Esta consideración facilita la solución del problema, ya que se evita el uso de técnicas estocásticas que consideren incertidumbre, y representa adecuadamente la realidad dado que una disgregación de la carga en el secundario tendría implicaciones físicas y económicas. Por lo tanto, una codificación que represente todas las posibles combinaciones de conexión y que además permita identificar cambios indeseables de secuencia en cargas industriales es la siguiente:

Tabla 1. Codificación del problema de balance de fases.

\begin{tabular}{ccc}
\hline $\begin{array}{c}\text { Número } \\
(\mathbf{H})\end{array}$ & Valor & Secuencia \\
\hline 1 & ABC & Igual \\
2 & BCA & \\
3 & CAB & \\
4 & ACB & Cambia \\
5 & BAC & \\
6 & CBA & \\
\hline
\end{tabular}

La conexión inicial que se asume para todas las cargas corresponde a $H=1$, por lo cual una alternativa de solución que sugiera una conexión $\mathrm{ABC}$ indica que no hay cambio alguno, mientras que las demás configuraciones implican un cambio con o sin modificación de la secuencia. Por ejemplo la configuración $\mathrm{H}=6$ (CBA) establece una conexión como se muestra en la figura 2 , donde de la conexión inicial $\mathrm{ABC}$ se cambia a la conexión CBA. Las primeras tres configuraciones conservan la secuencia de la carga, lo cual es especialmente importante en sistemas industriales en donde la presencia de motores eléctricos hace indeseable realizar cambios en la secuencia y las posibles combinaciones que se pueden tener en cuenta corresponden a $\mathrm{H}=1,2,3$. La explosión combinatorial del problema existe, fundamentalmente, porque el cambio en la configuración de cualquiera de las cargas del sistema puede ocasionar un aumento o disminución en las pérdidas del sistema.

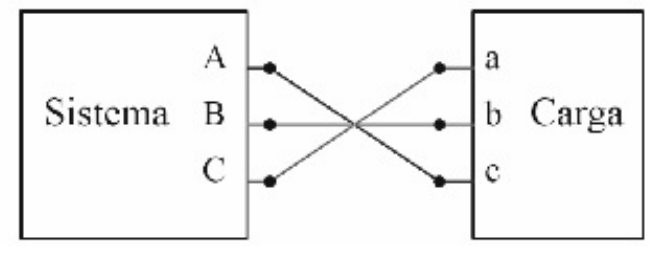

Figura 2: Ejemplo de codificación.

Cabe anotar que los casos especiales de interconexión como son alimentadores monofásicos, los cuales no permiten modificaciones en la conexión de la carga, y alimentadores trifásicos con cargas monofásicas son manejados como restricciones e incorporados en el algoritmo como casos especiales. Así por ejemplo, si se encuentra un alimentador trifásico $\mathrm{ABC}$ con una carga monofásica conectada entre las fases $\mathrm{AB}$, ésta sólo podrá reconectarse entre las fases BC ó AC.

\subsection{Criterio de Sensibilidad}

El grado de desbalance de un nodo puede ser expresado mediante diferentes tipos de índices los cuales pueden basarse en componentes simétricas, corrientes promedio y corrientes de secuencia, entre otros. En este trabajo se ha elegido un índice de desbalance de fácil implementación que ha mostrado resultados aceptables dentro del proceso de optimización, el cual se define como:

$\delta=\frac{\operatorname{Max}\left(\left|I_{a}-I_{\text {prom }}\right|,\left|I_{b}-I_{\text {prom }}\right|,\left|I_{c}-I_{\text {prom }}\right|\right)}{I_{\text {prom }}} \cdot 100 \%$

Donde la Iprom representa la corriente promedio expresada como:

$$
\text { Iprom }=\frac{I_{a}+I_{b}+I_{c}}{3}
$$

Se puede observar que el valor de este índice es cero cuando existe balance total y sirve tanto para guiar el proceso de optimización, de forma que la convergencia sea más rápida, como para indicar la calidad de una solución. También puede ser implementado igualmente con voltajes, pero se prefiere el uso de corrientes debido a que éstas son más sensibles al desbalance. 


\section{Descripción de la técnica combinatorial colonia de hormigas.}

Una metaheurística relativamente reciente es la Optimización basada en Colonias de Hormigas (ACO: Ant Colony Optimization), la cual se inspira en el comportamiento que rige a las hormigas de diversas especies para encontrar los caminos más cortos entre las fuentes de comida y el hormiguero [5]. Desde el trabajo inicial de Dorigo [6], Maniezzo y Colorni, la ACO se ha convertido en un campo de investigación importante y un gran número de autores han desarrollado modelos cada vez más sofisticados para solucionar de manera satisfactoria un gran número de problemas de optimización combinatorial. Igualmente se ha incrementado el número de desarrollos teóricos sobre los algoritmos que se proponen. Las hormigas son insectos sociales que viven en colonias y que, debido a su colaboración mutua, son capaces de mostrar comportamientos complejos y realizar tareas difíciles desde el punto de vista de una hormiga individual. Un aspecto interesante del comportamiento de muchas especies de hormigas es su habilidad para encontrar los caminos más cortos entre su hormiguero y las fuentes de alimento. Este hecho es especialmente interesante si se tiene en cuenta que muchas de las especies de hormigas son casi ciegas, y no existe la posibilidad de utilizar pistas visuales. Mientras que se mueven entre el hormiguero y la fuente de alimento, algunas especies de hormigas depositan una sustancia química denominada feromona. Si no se encuentra ningún rastro de feromona, las hormigas se mueven de manera aleatoria, pero cuando hay presencia de feromona depositada en el terreno tienen mayor tendencia a seguir el rastro, de esta forma se establece un medio de comunicación que hace que el comportamiento global del hormiguero tenga características inteligentes de optimización. Las hormigas prefieren de manera probabilística los caminos marcados con una concentración superior de feromona. Cuanto más fuerte es el rastro de feromona, mayor es la probabilidad de elegirlo. Puesto que las hormigas depositan feromona en el camino que siguen, este comportamiento lleva a un proceso de autorrefuerzo que concluye con la formación de rastros señalados por una concentración de feromona elevada. Inicialmente no existe ningún rastro de feromona en el medio, cuando una hormiga llega a una intersección, elige de manera aleatoria una de las bifurcaciones posibles. Según transcurre el tiempo y mientras las hormigas están recorriendo los caminos más prometedores, estos van recibiendo una cantidad superior de feromona. Este proceso hace que la probabilidad de que una hormiga escoja el camino más corto aumente progresivamente hasta que el recorrido de la colonia converja al más corto de todos los caminos posibles. Esta convergencia se complementa con la acción del entorno natural que provoca que la feromona se evapore transcurrido un cierto tiempo. Así, los caminos menos prometedores pierden progresivamente feromona porque son visitados cada vez por menos hormigas. Los algoritmos de ACO son esencialmente algoritmos constructivos: en cada iteración del algoritmo, cada hormiga construye una solución al problema recorriendo un grafo de construcción. Cada arista del grafo, que representa los posibles pasos que la hormiga puede dar, tiene asociada dos tipos de información que guían el movimiento de la hormiga: Información heurística, que mide la preferencia heurística de moverse desde un nodo a otro (exploración). Información de los rastros de feromona artificiales, que mide la "deseabilidad aprendida" del movimiento de un nodo a otro (explotación).

\section{Balance de fases usando colonia de hormigas}

Basado en la metodología de colonia de hormigas, se desarrolla el algoritmo de balance de fases en el cual se modela el rastro químico como una matriz de feromonas $(F)$ de tamaño $6 \times \mathrm{Nc}$, donde $\mathrm{Nc}$ es el número de cargas del sistema. Cada elemento Fij representa un nivel promedio de mejoría de las pérdidas cuando se modifica la carga $j$ implementando la configuración i. Este valor es actualizado en cada iteración de acuerdo al índice de desbalance y a las pérdidas totales como se muestra en la expresión (10). Cada hormiga se modela por una función matemática que determina una configuración de las cargas de acuerdo a dos criterios:

$\checkmark$ Exploración: Se elige de forma aleatoria una de las seis posibles configuraciones de la carga teniendo en cuenta una distribución de probabilidades dada por la matriz de feromonas. 
$\checkmark$ Explotación: Se elige la configuración que presente mayor contenido de feromona.

La función de cada hormiga consiste en recorrer cada una de las cargas y establecer su conexión de acuerdo a uno de estos dos criterios escogido con base en una regla probabilística de acuerdo a un parámetro de exploración IE definido previamente según un conocimiento previo del problema o realizando algunas pruebas de calibración. El parámetro IE corresponde a un valor definido en el intervalo abierto $(0,1)$, de manera que si, por ejemplo, $\mathrm{IE}=0.5$ existe la misma probabilidad de realizar exploración o explotación. Por otra parte, un valor de IE cercano a cero implica una escasa probabilidad de realizar exploración y una muy alta probabilidad de realizar explotación, por lo cual sería un procedimiento altamente determinístico que podría conducir, eventualmente, a una convergencia temprana a un óptimo local. Un valor de IE cercano a uno proporciona mayor aleatoriedad al proceso lo cual, eventualmente, podría ocasionar un mayor esfuerzo computacional. En caso que se realice explotación en un nodo determinado, se selecciona la configuración con mayor valor de feromona, en caso contrario se elige la configuración usando selección por ruleta, donde cada porción de la misma es obtenida usando la siguiente expresión:

Para ilustrar el proceso de selección, supóngase una ruleta como la mostrada en la figura 1, donde cada porción se obtuvo usando la expresión (9).

$$
P(i, j)=\frac{\operatorname{Feromona}(i, j)}{\sum_{k}^{6} \operatorname{Feromona}(i, k)}
$$

Posteriormente, se genera un número aleatorio entre 0 y $100 \%$ el cual permite ubicar la porción de la ruleta correspondiente, teniendo en cuenta que cada porción corresponde a una de las 6 posibles configuraciones. $\mathrm{Si}$, por ejemplo, el número aleatorio es 68.5, la configuración seleccionada sería la $\mathrm{H}=5$.

Esta metodología puede ser vista como una forma de combinar aleatoriamente los conceptos de exploración del espacio de solución con el de explotación de los rastros de feromona. Las alternativas con mayor área poseen mayor probabilidad de ser elegidos, inclinando la búsqueda

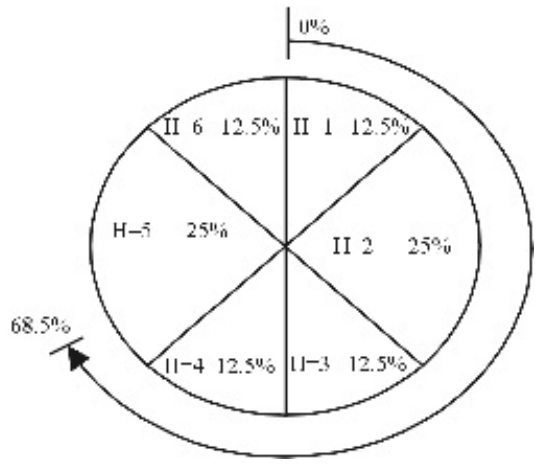

Figura 3: Selección de configuraciones por ruleta.

en la explotación de estos caminos; sin embargo, la naturaleza aleatoria del procedimiento alberga la probabilidad de escoger alternativas con probabilidad baja, obligando al algoritmo a explorar por caminos transitados con poca frecuencia.

En cada recorrido puede ocurrir que la solución elegida sea no factible al violar la restricción de cambio de secuencia, en este caso la nueva configuración deberá ser factibilizada mediante un proceso consistente en restar 3 al valor de $\mathrm{Hi}$ para terminar en una configuración que conserve la secuencia. En el caso de transformadores monofásicos el cambio debe realizarse en el primario. Una vez finalizado este recorrido se procede a calcular la función objetivo mediante el flujo de carga trifásico y si existe una reducción en las pérdidas que mejore la calidad de la mejor solución encontrada hasta el momento (incumbente), ésta es actualizada así como también la matriz de feromonas en todos los nodos, aún los nodos que presenten un aumento en el índice de desbalance. Esta actualización produce un aumento en los elementos de la matriz de feromonas que correspondan a la configuración actual, de forma que en las siguientes iteraciones dicha configuración tendrá mayor probabilidad de ser elegida. El valor de aumento (Dij ) en la matriz de feromonas es constante en este caso y es un parámetro de calibración definido previamente. Adicionalmente, existe el factor de evaporación (Ev), considerado como un factor de "Olvido" que proporciona al método mayor diversidad, evitando convergencias prematuras a óptimos locales. La evaporación de la feromona se aplica sobre cualquier tipo de configuración, aún si corresponde a una de buena calidad para el sistema; pero su efecto se 
acentúa con mayor énfasis en las configuraciones de poca calidad (caminos poco transitados). La ecuación de actualización de la matriz de feromona es la siguiente:

Si la nueva configuración, producto del recorrido de la hormiga, presenta una función objetivo de peor calidad que la incumbente, se actualizan sólo los

$F_{i j}=F_{i j} \cdot E_{v}+D_{i j}$

valores en los nodos en los cuales hay una reducción en el índice de desbalance. El procedimiento global continúa durante un número de iteraciones predeterminado y el número de hormigas de la colonia determina el número de recorridos globales por cada iteración. Si cumplido un número de iteraciones no se produce ningún cambio en la incumbente, se aplica un proceso de diversificación consistente en eliminar toda la información de los rastros de feromona contenida en la matriz y privilegiando en la nueva matriz de feromonas a aquellos nodos del sistema donde el índice de desbalance es inferior a 1 , lo cual se considera, en este caso, un desbalance moderado. Para privilegiar estos nodos se deposita en la posición correspondiente de la nueva matriz de feromonas una cantidad superior a la de los otros nodos. Algunos valores, para la matriz de feromona, que mostraron buen desempeño en el proceso de optimización son $F_{i j}=2$ para los nodos con índice de desbalance inferior a 1 y $F_{i j}=a$ para los demás nodos, donde $a$ es un aleatorio entre 0 y 1 . De esta forma el proceso es reiniciado conservando una memoria de las iteraciones anteriores. El algoritmo completo se describe en las figuras 4 y 5 .

La figura 4 muestra el algoritmo que representa el comportamiento individual de una hormiga de la colonia, esta función se ejecuta varias veces para lograr una solución óptima y es invocada por el algoritmo global en donde la matriz de feromonas es inicializada de forma aleatoria. El mayor esfuerzo computacional está dado por el flujo de carga trifásico, por lo cual el uso de un método de flujo de carga radial es recomendable ya que evita el cálculo de matrices jacobianas y la implementación de técnicas de dispersidad.

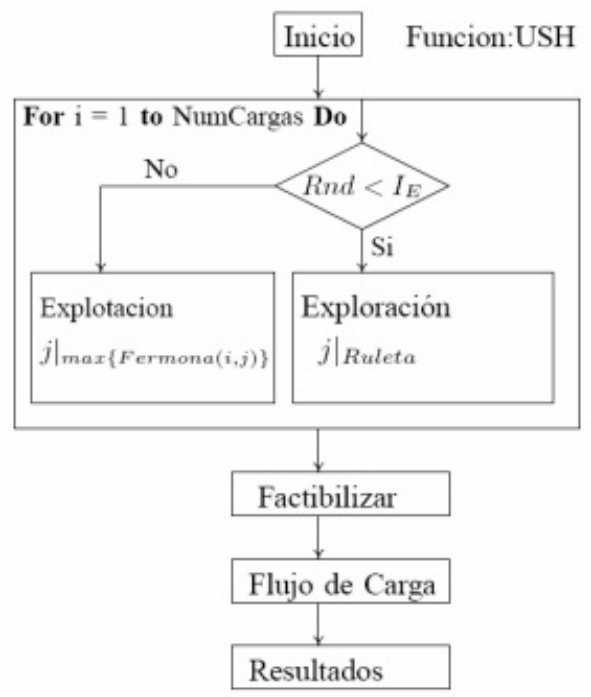

Figura 4: Proceso de optimización de una hormiga.

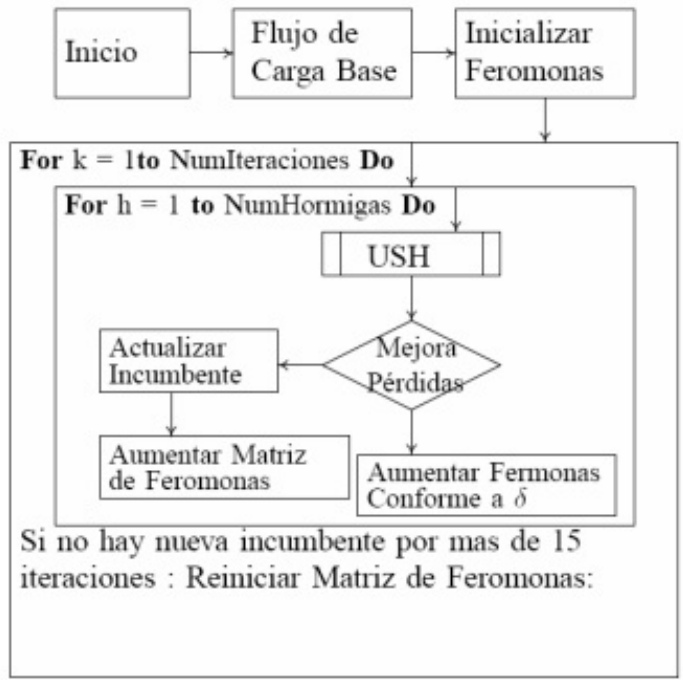

Figura 5: Proceso de optimización por colonia de hormigas

\section{Caso de prueba}

Se tomó como caso de prueba el sistema IEEE de 37 nodos disponible en [7] y que se muestra en la figura 5. Éste es un sistema real localizado en California constituido totalmente por líneas subterráneas y cargas de tipo residencial. Así mismo, contiene un transformador en condiciones desbalanceadas y un regulador en la subestación. Para facilitar el modelamiento del sistema no se incluye el regulador, 
por lo tanto se toma como nodo slack al nodo 701 con el valor reportado. El alto grado de desbalance de las cargas permite aplicar el algoritmo de balance de fases propuesto.

El sistema original es calculado para un punto de operación en la curva de carga. Los resultados son los siguientes: La reducción en pérdidas es de $9.15 \%$ que equivalen a $3,05 \mathrm{~kW}$, las nuevas configuraciones de carga se muestran en la siguiente tabla:

Tabla 2. Resultados del balance de fases.

\begin{tabular}{ccc}
\hline Parámetro & $\begin{array}{c}\text { Sistema } \\
\text { ORIGINAL }\end{array}$ & $\begin{array}{c}\text { Sistema } \\
\text { Modificado }\end{array}$ \\
\hline $\begin{array}{c}\text { Pérdidas de Potencia activa } \\
(\mathrm{kW})\end{array}$ & 33,29 & 30,24 \\
$\begin{array}{c}\text { Pérdidas de potencia reactiva } \\
(\mathrm{kVAr})\end{array}$ & 25,95 & 23,75 \\
\hline
\end{tabular}

Aunque en algunos nodos no se produjo un cambio en el índice de desbalance el efecto es visible de forma global dado que las pérdidas totales del sistema disminuyeron $9.15 \%$ y el nodo slack que inicialmente presenta un índice de desbalance alto pasa a tener uno menor. Aunque el nodo Slack no presenta carga conectada directamente, su grado de desbalance afecta considerablemente las pérdidas totales del sistema.

Algunos nodos como el 714 pasan a tener un mayor grado de desbalance demostrando que el algoritmo hace una optimización global en la que se sacrifica el índice de desbalance de algunos nodos particulares con el propósito de mejorar la configuración total.

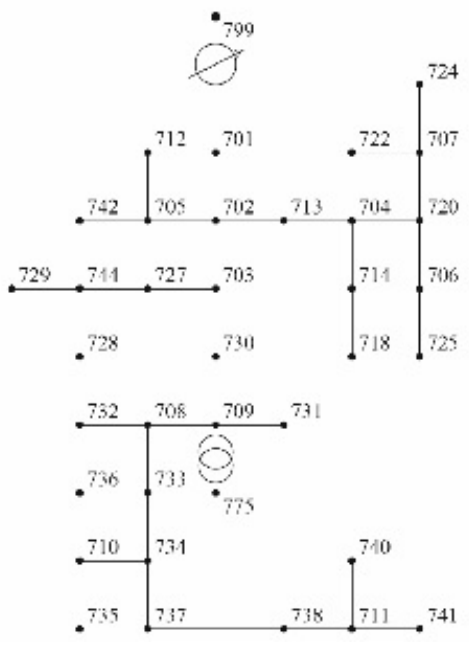

Figura 5: Ejemplo IEEE de 37Nodos
Tabla 3. Resultado configuraciones de carga.

\begin{tabular}{llll}
\hline Nodo & Cambio & $\begin{array}{l}\ddot{A} \\
(\%)\end{array}$ & $\begin{array}{l}\text { BASE } \\
(\%)\end{array}$ \\
\hline 701 & - & 16,93 & 3,19 \\
712 & - & 100,00 & 100,00 \\
713 & ACB & 19,47 & 13,49 \\
714 & CAB & 73,02 & 78,56 \\
718 & CBA & 100,00 & 100,00 \\
720 & BCA & 49,28 & 19,48 \\
722 & BAC & 80,09 & 79,83 \\
724 & CAB & 100,00 & 100,00 \\
725 & - & 100,00 & 100,00 \\
727 & CAB & 27,97 & 24,97 \\
728 & CAB & 0,01 & 0,01 \\
729 & - & 100,00 & 100,00 \\
730 & ACB & 25,34 & 10,32 \\
731 & BCA & 100,00 & 100,00 \\
732 & CAB & 100,00 & 100,00 \\
733 & CAB & 34,15 & 10,46 \\
734 & CBA & 33,07 & 10,05 \\
735 & - & 100,00 & 100,00 \\
736 & BCA & 100,00 & 100,00 \\
737 & CBA & 48,29 & 16,80 \\
738 & CAB & 39,33 & 47,56 \\
740 & CAB & 100,00 & 100,00 \\
741 & ACB & 100,00 & 100,00 \\
742 & BAC & 86,89 & 86,51 \\
744 & CAB & 41,68 & 18,76 \\
\hline
\end{tabular}

Los parámetros de la metodología son mostrados en la tabla 4.

Tabla 4. Parámetros de la metodología de colonia de hormigas.

\begin{tabular}{|c|c|}
\hline Parámetro & Valor \\
\hline Número de hormigas & 10 \\
\hline Numero de iteraciones & 30 \\
\hline Índice de Exploración & 0,8 \\
\hline Nivel de evaporación & 0,7 \\
\hline $\begin{array}{l}\text { Número máximo de } \\
\text { cambios }\end{array}$ & 25 \\
\hline $\begin{array}{l}\text { Permitir cambio de } \\
\text { secuencia }\end{array}$ & $\mathrm{Si}$ \\
\hline
\end{tabular}

El tiempo de cálculo es de 15 segundos, utilizando un computador con procesador Intel Pentium 4 de 2,66 Ghz.

Los resultados fueron corroborados con la metodología de búsqueda Tabú con tiempos de cálculo similares[11]. 


\section{Conclusiones y recomendaciones}

$\checkmark$ El balance de fases permite reducir el nivel de pérdidas del sistema con un costo de inversión mínimo, por lo cual implementar programas de balanceo de fases en las empresas de distribución de energía eléctrica es una alternativa viable, que debe ser considerada no sólo para la reducción de pérdidas técnicas, sino también para aumentar la calidad, seguridad y confiabilidad del servicio, así como para reducir las inversiones adicionales en la aplicación de otras medidas correctivas posteriores tales como la reconfiguración y la compensación de reactivos.

Los resultados obtenidos muestran que el método de optimización utilizado tiene en cuenta el comportamiento global del sistema ante cambios puntuales en las conexiones de las cargas en los nodos. Esto se evidencia al presentarse algunos nodos con empeoramiento en el índice de desbalance pero que, desde el punto de vista global, ayudan a reducir en mayor porcentaje las pérdidas del sistema.

$\checkmark$ El índice de desbalance del nodo Slack generalmente presenta una importante disminución cuando el sistema es sometido al procedimiento de balanceo. Este fenómeno es de fácil explicación si se considera que la potencia inyectada al sistema es directamente proporcional a las pérdidas totales del mismo, por lo cual un balance en el nodo Slack afecta la totalidad del sistema.

$\checkmark$ El problema de balance de fases propuesto utiliza un modelo que tiene en cuenta la curva de carga para un período de tiempo determinado. Esto es de gran importancia en países con sistemas eléctricos con escasos recursos económicos y pobremente automatizados, ya que los cambios sugeridos son efectivos para todo el período de estudio.

$\checkmark$ La metodología de colonia de hormigas se muestra como una alternativa importante que puede ser fácilmente adaptada a la solución de problemas combinatoriales en ingeniería eléctrica. Muchas modificaciones pueden ser propuestas para mejorar el desempeño del algoritmo, tal es el caso de las técnicas híbridas que combinan las mejores características de diferentes métodos de optimización. $\checkmark$ En este trabajo se muestra un modelo aplicable al caso colombiano en el cual se tienen en cuenta restricciones como el cambio de secuencia y se aplica una metodología combinatorial relativamente nueva en aplicaciones de ingeniería eléctrica donde ésta se modifica teniendo en cuenta las particularidades del problema.

$\checkmark$ Como recomendación general, se puede profundizar en el estudio de diferentes métodos de reducción de pérdidas técnicas proyectados en un horizonte de tiempo y abordados con diferentes y novedosas técnicas de optimización combinatorial como son: colonia de hormigas, partículas swarm, algoritmos genéticos, búsqueda tabú, simulated annealing, entre otras. Actualmente, el grupo de planeamiento eléctrico de la Universidad Tecnológica de Pereira viene desarrollando una investigación sobre reducción de pérdidas técnicas usando estas metodologías en un contexto integrado por etapas con el auspicio de ISA y COLCIENCIAS que ha mostrado resultados prometedores en la aplicación de estas técnicas a problemas propios de la ingeniería eléctrica.

\section{Agradecimientos}

Los autores expresan sus agradecimientos al grupo de planeamiento en sistemas eléctricos de la Universidad Tecnológica de Pereira y al Grupo de Desarrollo en Investigación Operativa DINOP por su apoyo.

\section{Referencias bibliográficas}

[1] GO“ NEN, T. "Electric Power Distribution System Engineering". McGraw Hill Editions. 1987. 739p.

[2] Ruiz, A., Echeverrí-Granada, M., Gallego-Rendón, R. A. "Flujo de carga trifásico para sistemas radiales de distribución". Scientia Et Technica. Pereira: , v.22, 2004

[3] Zhu, J., Griff, B., and Chow, M., "Phase balancing using Mixed-Integer programming". IEEE Trans. Power Systems. Vol 13. No 4. Nov 1998. pp 1487 1492.

[4] "Phase balancing using Simulated Annealing". IEEE Trans. Power Systems. Vol 14 No 4. Nov 1999. Pp 1508-1513. 
[5] Lin, Ch. et al. "Heuristic rule-based phase balancing of distribution systems by considering customer load patterns". IEEE Trans. Power Systems. Vol 20 N 2. May 2005. pp 709-716.

[6] Díaz, A. et al. "Optimización Heurística y Redes Neuronales”. Editorial Paraninfo. España 1996.

[7] Doringo, M. et al. "The Ant System: Optimization by a colony of cooperating agents". IEEE Transactions on Systems, Man, and Cybernetics-Part B, Vol 26, No 1,1996, p.1-13.

[8] http://ewh.ieee.org/soc/pes/dsacom/testfeeders.html

[9] Kochenberger, G A, and Glover F. "Handbook of metaheuristics". Kluwer academic publishers, 1 edition, 2003.

[10] Maniezzo, V., Doringo, M., and Colorni, A. "The ant system: Optimization by a colony of cooperating agents". IEEE Trans. on Systems, Man, and Cybernetics Part B,,26(1):113, Nov. 1996.

[11] Gallego, R., Galvis C., Granada, M. Balance de fases por búsqueda tabú. Revista de la Escuela Colombiana de Ingeniería. (en evaluación). 